\title{
The Fusion Protein of CTP-HBcAg18-27-Tapasin Mediates the Apoptosis of CD8+T Cells and CD8+T Cell Response in HLA-A2 Transgenic Mice
}

\author{
Yu-Yan Tang ${ }^{1}$; Zheng-Hao Tang ${ }^{1}$; Yi Zhang ${ }^{1}$; Meng Zhuo ${ }^{1}$; Guo-Qing Zang ${ }^{1}$; Xiao-Hua Chen ${ }^{1, *}$; \\ Yong-Sheng $\mathrm{Yu}^{1,}$ \\ ${ }^{1}$ Department of Infectious Disease, Shanghai Jiao Tong University Affiliated Sixth People's Hospital, Shanghai, China \\ *Corresponding Authors: Xiao-Hua Chen, Department of Infectious Disease, Shanghai Jiao Tong University Affiliated Sixth People's Hospital, 200233 Shanghai, China. Tel/Fax: +86- \\ 2164369181, E-mail: chenxiaohua2000@163.com; Yong-Sheng Yu, Department of Infectious Disease, Shanghai Jiao Tong University Affiliated Sixth People's Hospital, 200233 Shang- \\ hai, China. Tel/Fax:+86-2164369181, E-mail: yuyongsheng@medmail.com.cn
}

Received: November 13, 2013; Revised: January 20, 2014; Accepted: February 2, 2014

\begin{abstract}
Background: HBV-specific cytotoxic T lymphocyte (CTL) activity is believed to play a critical role in controlling HBV infection. The phosphatidylinositol 3-kinase (PI3K)/Akt signaling pathway manipulates cell fate decisions in many different cell types by regulating the activity of downstream effectors. We have previously testified that the fusion protein of CTP-HBcAg18-27-Tapasin could enter the cytoplasm of dendritic cells and efficiently induce robust specific CTL response in vitro.

Objectives: In the present study, we evaluated specific CTL response and the level of apoptosis of CD8+ T cells induced by CTP-HBcAg18-27Tapasin in HLA-A2 transgenic mice (H-2Kb). Meanwhile, we preliminary investigated PI3K, phosphorylation level of Akt, and mammalian target of rapamycin (mTOR) as positive regulator of the magnitude and effector function of the hepatitis B virus-specific cytotoxic $\mathrm{T}$ lymphocytes in HLA-A2 transgenic mice.

Materials and Methods: HLA-A2 transgenic mice were immunized by intramuscular injection in the hind legs three times at one-week intervals with PBS, CTP-HBcAg18-27-Tapasin ( $50 \mu \mathrm{g})$, CTP-HBcAg18-27 (50 $\mu \mathrm{g})$, HBcAg18-27-Tapasin ( $50 \mu \mathrm{g})$, and HBcAg18-27 (50 $\mu$ g). One week after the last immunization, mice were sacrificed and splenocytes were harvested in strile condition. The specific CTL response was analyzed by flow cytometry and enzyme linked immunosorbent assay (ELISA); the expression of (PI3K)/Akt signaling was detected by RTPCR and western blot.

Results: The results showed that CTP-HBcAg18-27-Tapasin significantly increased the percentages of IFN- $\gamma^{+} \mathrm{CD} 8 \alpha^{+} \mathrm{T}$ cells, the numbers of these polyfunctional triple-cytokine-producing (IFN- $\gamma$, TNF- $\alpha$, and IL-2) CD8 ${ }^{+}$T cells, the secretion of cytokine IFN- $\gamma$, IL-2, and TNF- $\alpha$, while in comparison to control group, it significantly decreased the percentage of apoptotic CD8 ${ }^{+} \mathrm{T}$ cells in HLA-A2 transgenic mice. Moreover, the expression of PI3K, P-Akt, and P-mTOR was significantly upregulated in CTP-HBcAg18-27-Tapasin group compared with control groups. Conclusions: In conclusion, CTP-HBcAg18-27-Tapasin could reduce apoptosis of $\mathrm{CD}^{+}{ }^{+} \mathrm{T}$ cells, increase the percentages of IFN- $\gamma^{+} \mathrm{CD} 8 \alpha^{+}$ T cells, and elicit cell-mediated immunity in HLA-A2 transgenic mice; these processes were associated with activation of the PI3K/Akt signaling pathway.
\end{abstract}

Keywords: Tapasin; Mice, Transgenic; T-Lymphocytes, Cytotoxic; PI3K/Akt

\section{Background}

Studies of chronic infections with viruses such as hepatitis B, hepatitis C, and HIV indicate that persistent antigen stimulation induces peripheral $\mathrm{T}$ cell tolerance; virus-specific cytotoxic T lymphocyte (CTL) either suffer clonal deletion or lose their functions, a condition termed immunologic tolerance $(1,2)$. Common denominator underlying antigenic stimulation persistence in these chronic B virus infections ( $\mathrm{CHB}$ ) is the dysregulation of virus-specific $\mathrm{T}$ cell responses (35). During $\mathrm{CHB}$, the abundance of virus-specific $\mathrm{CD}^{+} \mathrm{T}$ cells is controlled by the balance between these cellular processes that a continuum of $\mathrm{T}$ cell proliferation and apoptosis (6-8). However, HBV-specific cytotoxic $\mathrm{T}$ lymphocyte (CTL) activity might play an important role in HBV clearance, because the magnitude of the $\mathrm{CD}^{+} \mathrm{T}$ cell response has a key role in determining the efficiency of viral control (7).

HBV core 18-27 peptide (HBcAg18-27) is recognized as the most efficient agent that primes the human leukocyte antigen (HLA) class-I-restricted immune response in acutely infected patients $(9,10)$. The HLA-A2 transgenic mice used in the experiments express heterodimeric HLA-A2.1/Kb molecules in the context of a background of $\mathrm{H}-2$ class I molecules (11). HBcAg18-27 is also immunodominant in the context of HLA-A2.1. Previous studies suggest that Tapasin, an endoplasmic

Implication for health policy/practice/research/medical education:

This strategy might have a therapeutic value that can be a promising therapeutic strategy for hepatitis B virus clearance in patients with chronic HBV, and a promising HBV vaccine for preventing HBV infection.

Copyright (c) 2014, Kowsar Corp.; Published by Kowsar Corp. This is an open-access article distributed under the terms of the Creative Commons Attribution License, which permits unrestricted use, distribution, and reproduction in any medium, provided the original work is properly cited. 
reticulum (ER) chaperone, stabilizes the peptide-receptive MHC I conformation, allowing peptide exchange and greater peptide translocation into the ER, which enhances specific MHC class I-restricted CTL activity (12-14). Thus, combining the specificity of CTL epitope (HBcAg18-27), chaperone Tapasin, and transfer by the cell-penetrating property of cytoplasmic transduction peptide (CTP), may elicit a robust specific CTLs response. We have previously testified that the fusion protein CTP-HBcAg18-27-Tapasin could enter the cytoplasm of dendritic cells, and efficiently induce robust specific CTL response, in vitro $(15,16)$.

Mammalian target of rapamycin (mTOR) is a key intermediary in multiple mitogenic signaling pathways and plays a central role in modulating proliferation and angiogenesis in normal tissues and neoplastic processes (17). The PI3K pathway translates numerous extracellular stimuli into a wide range of essential cellular processes through 3-phosphoinositide-dependent effectors such as the serine/threonine kinase Akt. Some Studies previously reported that PI3K is strongly activated in naive $\mathrm{T}$ cells after Ag recognition (18-21). During $\mathrm{CHB}$, the abundance of virus-specific $\mathrm{CD}^{+} \mathrm{T}$ cells is controlled by the balance between these cellular processes that a continuum of $\mathrm{T}$ cell proliferation and apoptosis (6-8). Thus, the PI3K/Akt signaling pathway might be involved in polarization towards $\mathrm{CD} 8^{+} \mathrm{T}$ cells.

\section{Objectives}

In the present study, we evaluated specific CTL response and the level of apoptosis of $\mathrm{CD}^{+} \mathrm{T}$ cells induced by CTP-HBcAg18-27-Tapasin in HLA-A2 transgenic mice $(\mathrm{H}-2 \mathrm{~Kb})$. Meanwhile, we preliminary investigated the PI3K, phosphorylation level of Akt, and mammalian target of rapamycin (mTOR) as positive regulators of the magnitude and effector function of the hepatitis $B$ virus-specific CTLs in HLA-A2 transgenic mice.

\section{Materials and Methods}

\subsection{Reagents, Mice and Fusion Proteins}

The fluorescent antibodies and the corresponding isotype controls were obtained from eBioscience (USA), and western blot antibodies were purchased from Abcam (Hong Kong). ELISA kits for IFN- $\gamma$, TNF- $\alpha$, and IL-2 was obtained from R\&D Co. Ltd. (USA). Ionomycin, monensin, and phorbol 12-myristate 13-acetate (PMA) were purchased from Sigma (USA). Soluble fusion proteins CTPHBcAg18-27-Tapasin, CTP-HBcAg18-27, HBcAg18-27-Tapasin, and HBcAg18-27 were maintained in our lab (16).

\subsection{Mice and Treatments}

HLA-A2 transgenic mice ( $\mathrm{H}-2 \mathrm{~Kb})$, six to eight weeks old, which had the murine $\beta 2$ microglo-bulin $(\beta 2 \mathrm{~m})$,
$\mathrm{H}-2 \mathrm{Db}$ genes knocked out, and were transgenic for a chimeric human HLA-A2.1 expressing the a1 and a2 domains of HLA-A2.1 and a mouse H-2Db-derived a3 domain to allow interaction with mouse CD8 (11), were purchased from The Jackson Laboratories and were maintained in the Shanghai Sixth People's Hospital Animal Centre under specific pathogen-free conditions. All experimental procedures were performed in accordance with approved protocols and regulations by the laboratory animal ethical commission of Shanghai Jiao Tong University. HLA-A2 transgenic mice were allocated into five groups with six mice in each group. Mice were immunized by intramuscular injection of PBS, CTPHBcAg18-27-Tapasin $(50 \mu \mathrm{g}), \mathrm{CTP}-\mathrm{HBcAg} 18-27(50 \mu \mathrm{g})$, HBcAg18-27-Tapasin $(50 \mu \mathrm{g})$, and HBcAg18-27 $(50 \mu \mathrm{g})$ in the hind legs three times at one-week intervals. In our preliminary study, we also used the doses of $20 \mu \mathrm{g}$ and $100 \mu \mathrm{g}$. We found that the dose of $50 \mu \mathrm{g}$ was the most appropriate dose for our purpose (data not shown). One week after the last immunization, mice were sacrificed and splenocytes were harvested for this experiment in aseptic condition.

\subsection{Cell Isolation}

HLA-A2 transgenic splenocytes were collected and treated with lysis buffer to eliminate red blood cells, washed, and re-suspended in RPMI-1640 (Giboco BRL) with $10 \%$ FBS (Giboco BRL). Lymphocytes were derived from splenocytes using nylon wool columns (Wako, Japan). Single-cell suspensions of lymphocytes $\left(2 \times 10^{6}\right.$ cells/well) were grown in six-well plates (Corning). The purities of the isolated $\mathrm{T}$ cells were determined by flow cytometry analysis after staining with anti-CD3- PE-Cy5 (eBioscience, United States), and the samples with purity of more than $80 \%$ were used for this experiment.

\subsection{Measurement of Function of CD8+T Cells by Intracellular Cytokine Staining (ICCS)}

To investigate the number of IFN- $\gamma$ secreting cells and also production of TNF- $\alpha$ and IL- 2 by the immunized mouse $\mathrm{T}$ cells, $\mathrm{T}$ lymphocytes $\left(1 \times 10^{6}\right.$ cells $\left./ \mathrm{mL}\right)$ collected from immunized mice were analyzed by flow cytometry. The T lymphocytes were stimulated in the presence of $10 \mu \mathrm{g} / \mathrm{mL}$ HBcAg18-27 for six hours. After incubation for three hours, ionomycin $(1 \mu \mathrm{g} / \mathrm{mL})$, monensin $(1.7 \mu \mathrm{g} / \mathrm{mL})$, and PMA $(25 \mu \mathrm{g} / \mathrm{mL})$ (15) were added and incubation continued for another three hours. After incubation, the wells were washed twice with PBS; cells were then incubated with saturating concentrations of PE conjugated anti-CD8 $\alpha$ McAb. After permeabilization with Fix and Perm reagent A and B (BD Biosciences, USA), the cells was stained with FITC-labeled anti-interferon- $\gamma$ (IFN- $\gamma$ ) McAb, APC conjugated anti-IL-2 $\mathrm{McAb}$, and PE-CY7- labeled anti-TNF- $\alpha$ for 20 minutes. Af- 
ter two washes, the cells were analyzed by flow cytometry (COULTER EPICS XL Flow Cytometer (Beckman)).

\subsection{Cytokines Release Assay}

T cells $\left(2 \times 10^{6}\right.$ cells $\left./ \mathrm{mL}\right)$ from the HLA-A2 transgenic mice harvested from immunized mice were incubated in 24-well plates at $37^{\circ} \mathrm{C}$ in the presence of $10 \mu \mathrm{g} / \mathrm{mL} \mathrm{HB}$ cAg18-27. After 72 hours of incubation, culture supernatants were harvested and the level of cytokines including IFN- $\gamma$, TNF- $\alpha$ and IL- 2 were analyzed by ELISA kits according to the manufacturer's protocol. The concentrations of cytokines in the samples were determined from the standard curves. Data are expressed as $\mathrm{pg} / \mathrm{mL}$.

\subsection{Assessment of Apoptosis Ex Vivo}

T cells $\left(2 \times 10^{6}\right.$ cells $\left./ \mathrm{mL}\right)$ from harvested spleens of immunized mice were cultured in six-well plates at $37^{\circ} \mathrm{C}$ as described above, except that no red blood cell lysis was performed. After two washes with PBS, cells were incubated with APC-labeled anti-CD8 $\alpha$ McAb. Annexin V-FITC and Propidium Iodide (PI) staining (Invitrogen, USA) were then performed according to the manufacturer's instructions. The whole cell population of thrice stained positive cells among antigen-specific CD8+ T cells was analyzed by flow cytometry.

\subsection{Real-Time PCR}

T cells $\left(2 \times 10^{6}\right.$ cells $\left./ \mathrm{mL}\right)$ from spleens harvested from immunized mice were cultured in six-well plates at $37^{\circ} \mathrm{C}$. Next, cells were collected for total RNA isolation according to the protocol for Trizol Reagent (Invitrogen, USA). cDNA was generated using PrimeScript $1^{\text {st }}$ Strand cDNA Synthesis Kit (TaKaRa, Japan). Primers were designed by Primer Premier 5.0 according to the mRNA sequences of PI3K, Akt, and mTOR genes retrieved from GenBank, and synthesized by Sangon Biotech (Shanghai) Co., Ltd., China. The Primer sequences are shown in Table 1. Realtime PCR was performed using SYBRßPremix Ex TaqTM reagents (TaKaRa, Japan) on a LightCycler (Roche Diagnostic). PCR conditions were as follows: the thermal cycle parameters were 30 seconds at $95^{\circ} \mathrm{C}$ followed by $40 \mathrm{cy}$ cles of $95^{\circ} \mathrm{C}$ for five seconds and $60{ }^{\circ} \mathrm{C}$ for 20 seconds. The amount of target was calculated by the following equation: $2^{-\Delta \Delta \mathrm{Ct}}$. Three parallel reactions of each sample and internal control were performed.

\subsection{Western Blot}

The cells described above were washed twice with PBS, gently dispersed into a single-cell suspension, and homogenised using RIPA lysis buffer (Beyotime Institute of Biotechnology, China). Protein concentrations were determined using the Pierce BCA Protein Assay Reagent kit (Rockford, United States). Homogenates were diluted to the desired protein concentration with
$2 \times$ SDS-PAGE loading buffer (Invitrogen). Samples were boiled and loaded onto the polyacrylamide mini-gels (Invitrogen) for electrophoresis. Proteins from the gels were transferred to Immobilon-PVDF membranes (Millipore Corp., Bedford, MA, USA) using a semi-dry apparatus (Bio-Rad, Hercules, CA, United States). A rabbit anti-mouse PI3K (1:1000), P-Akt (1:5000), and P-mTOR (1:1000) monoclonal antibody was used as the primary antibody and horseradish peroxidase-conjugated goat anti-rabbit immunoglobulin-G antibody was used as the secondary antibody. Values obtained were normalized based on density values of internal b-actin.

\subsection{Statistical Analysis}

Data were expressed as mean \pm SD and were analyzed by the SPSS v.16.0 software. One-way ANOVA and posthoc least significant difference (LSD) test were used to determine the statistical significance in comparison to the control. P-values of 0.05 or less were considered statistically significant.

\section{Results}

\subsection{CTP-HBcAg18-27-Tapasin Induces IFN- $\gamma$ - Producing CD8+ T Cells in the Spleen}

We measured the amount of IFN- $\gamma$-producing $\mathrm{CD}^{+} \mathrm{T}$ cells by flow cytometry. The doubly stained cells were the positive ones. As shown in Figure 1, the percentages of specific IFN- $\gamma^{+} \mathrm{CD}^{+} \mathrm{T}$ cells from CTP-HBcAg18-27-Tapasin group $(2.83 \pm 0.15 \%)$ were significantly higher than the percentage of CTP-HBcAg18-27 (1.33 \pm 0.31\%), HBcAg1827-Tapasin (0.87 $\pm 0.15 \%)$ HBcAg18-27 (0.80 $\pm 0.2 \%)$, and PBS $(0.53 \pm 0.25 \%)(\mathrm{P}<0.01)$. The results demonstrated that the delivery of Tapasin and HBcAg18-27 via CTP enhanced the generation of IFN $-\gamma^{+} \mathrm{CD} 8^{+} \mathrm{T}$ cells in vivo.

Table 1. The Primer Sequences for PI3K, Akt, mTOR, and $\beta$-ctin

\begin{tabular}{ll}
\hline Gene & Sequence (5' to 3') \\
\hline PI3K & \\
\hline Forward & TCGGTCTGTAGATGAGGC \\
\hline Reverse & CGGAGGAATGGATGAGGG \\
\hline Akt & \\
\hline Forward & G TCGTCGCCAAGGATGAGG \\
\hline Reverse & GGTCGTGGGTCTGGAATGA \\
\hline mTOR & \\
\hline Forward & GCCACCTGGTATGAGAAGC \\
\hline Reverse & CCAACACTGCCCTGTAAAA \\
\hline $\boldsymbol{\beta}$-ctin & \\
\hline Forward & CTCCATCCTGGCCTCGCTCG \\
\hline Reverse & GCTGTCACCTTCACCGTTCC \\
\hline
\end{tabular}


Tang Yet al.

\subsection{CTP-HBcAg18-27-Tapasin Enhances CD8+T Cell Function}

Next, we investigated whether the fusion protein of CTP-HBcAg18-27-Tapasin affected the effector function of $\mathrm{CD}^{+} \mathrm{T}$ cells. For this purpose, we used ELISA kits and ICCS to measure fusion protein induced production of cytokines (IFN- $\gamma$, TNF- $\alpha$, and IL-2). As shown in Figure 2 $\mathrm{A}, \mathrm{B}$, and $\mathrm{C}$, the number of IFN- $\gamma(703.44 \pm 21.01 \mathrm{pg} / \mathrm{mL})$, TNF- $\alpha(572.82 \pm 30.25 \mathrm{pg} / \mathrm{mL})$, and IL-2 (407.34 \pm 11.46 $\mathrm{pg} / \mathrm{mL}$ ) production were significantly greater in CTPHBcAg18-27-Tapasin group than in the CTP-HBcAg18-27
$(612 \pm 32.45,310.51 \pm 9.85$, and $403.63 \pm 32.25 \mathrm{pg} / \mathrm{mL}$ for IFN- $\gamma$, TNF- $\alpha$ and IL-2, respectively), HBcAg18-27-Tapasin, HBcAg18-27, and PBS groups. Notably, the numbers of these polyfunctional triple-cytokine-producing (IFN- $\gamma$, TNF- $\alpha$, and IL-2) CD8 ${ }^{+} \mathrm{T}$ cells in the CTP-HBcAg18-27-Tapasin group ( $0.72 \pm 0.10 \%)$ was higher than the control groups (Figure $2 \mathrm{D}$ ). The inability of $\mathrm{CD}^{+} \mathrm{T}$ cells to produce three cytokines is a hallmark of functional exhaustion $(22,23)$. Thus, our finding suggested that CTP-HBcAg18-27-Tapasin would enhance cytokine IFN- $\gamma$, TNF- $\alpha$, and IL-2 secretion, $C D 8^{+} \mathrm{T}$ cell function, and elicit cell-mediated immunity.

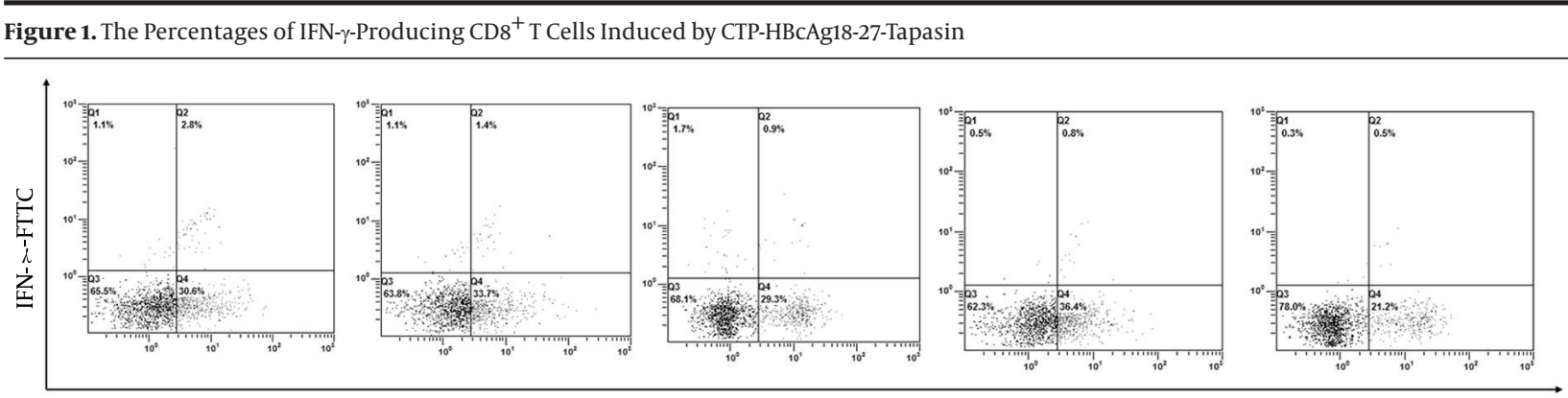

CD8- - -PE

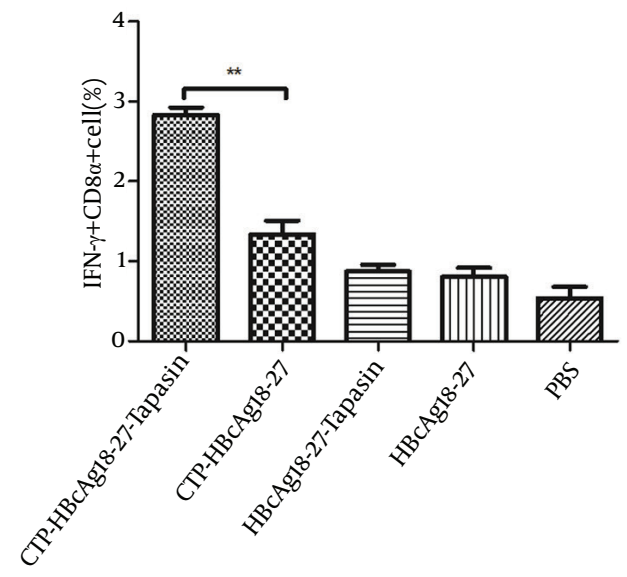

The whole cell population was analyzed by flow cytometry. CTP-HBcAg18-27-Tapasin enhanced a higher level of HBV-specific IFN- ${ }^{+} \mathrm{CD}^{+}{ }^{+} \mathrm{T}$ cells when compared to CTP-HBcAg18-27, HBcAg18-27-Tapasin, HBcAg18-27, and PBS. The data are presented as mean \pm SD from six mice from each group $\left({ }^{* *} \mathrm{P}<0.01\right)$. 
A

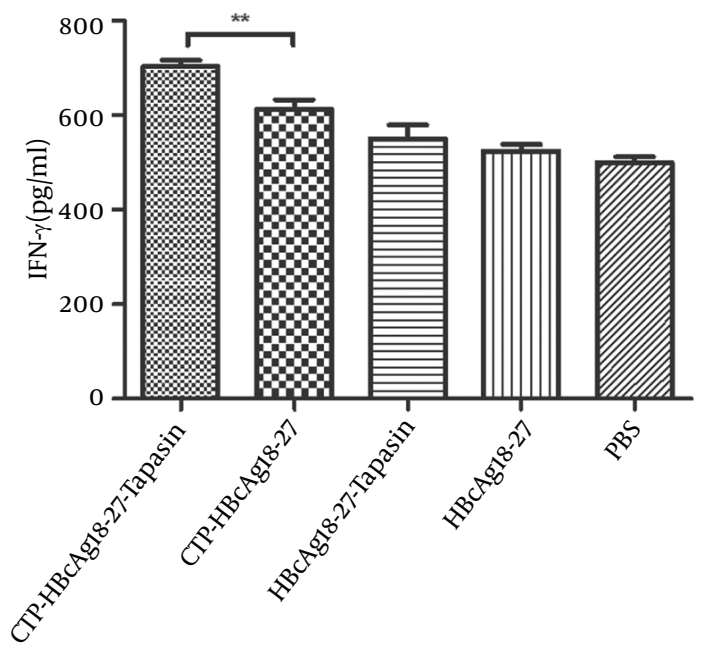

C

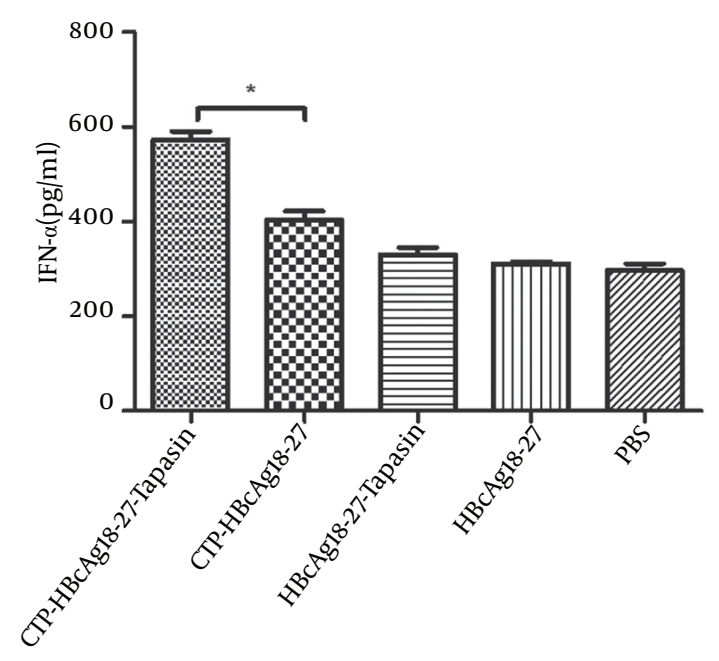

B

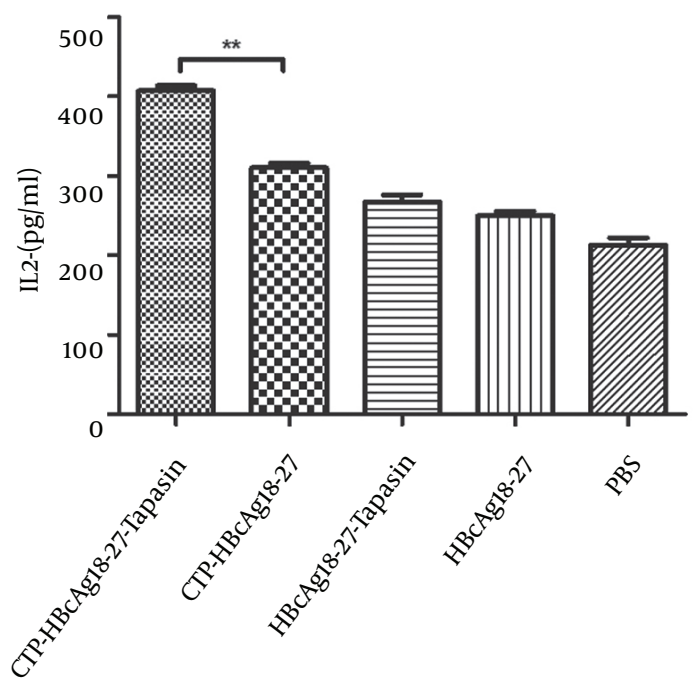

D

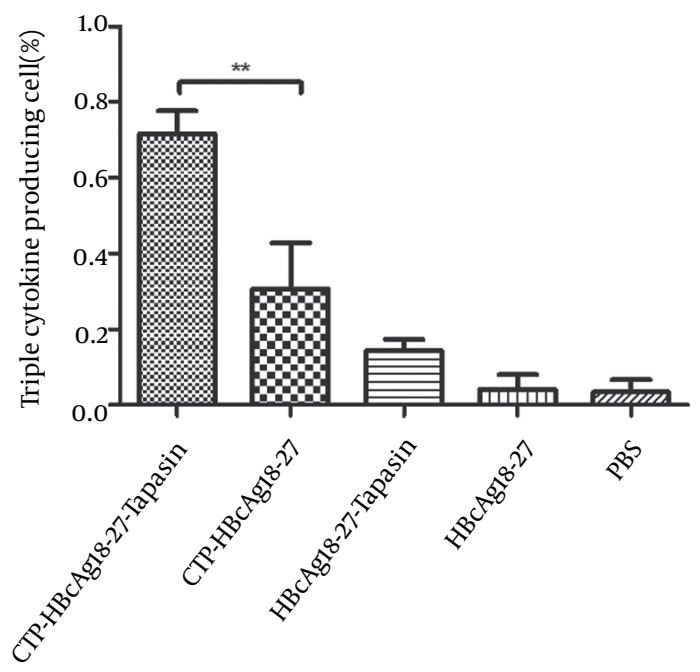

IFN- $\gamma$, TNF- $\alpha$, and IL-2 in CD8 ${ }^{+}$T cells. A, B, and C demonstrate that secretions of IFN- $\gamma$, TNF- $\alpha$, and IL-2 in the CTP-HBcAg18-27-Tapasin group were significantly higher than in the CTP-HBcAg18-27, HBcAg18-27-Tapasin, HBcAg18-27, or PBS groups. (D) The numbers of these polyfunctional triple-cytokine-producing $\left(\right.$ IFN- $\gamma$, TNF- $\alpha$, and IL-2) CD ${ }^{+}$T cells in CTP-HBcAg18-27-Tapasin group was higher than the control group. Data represent the mean \pm SD $(n=6)\left({ }^{*} \mathrm{P}<0.05\right.$, $\left.{ }^{* *} \mathrm{P}<0.01\right)$.

\subsection{Decreased Apoptosis of CD8+ T Cells Pulsed With CTP-HBcAg18-27-Tapasin}

The above results indicate that HBcAg18-27 via CTP transduction could efficiently induce $\mathrm{CD}^{+} \mathrm{T}$ cell response. However, the mechanism behind these results was not clear. During CHB, the abundance of virus-specific $\mathrm{CD}^{+} \mathrm{T}$ cells is controlled by the balance between these cellular processes, resulting in a continuum of T cell proliferation and apoptosis (6-8). Therefore, we further observed the level of apoptosis of $\mathrm{CD}^{+} \mathrm{T}$ cells by flow cytometry. The number of three stained positive cells was counted by flow cytometry. As shown in Figure 3, significantly lower percentages of apoptosis of $\mathrm{CD}^{+} \mathrm{T}$ cells were observed in mice immunized with CTP-HBcAg18-27-Tapasin (5.01 $\pm 0.56 \%$ ), compared to 
Tang Yet al.

CTP-HBcAg18-27 (16.30 \pm 5.96\%), HBcAg18-27-Tapasin (23 $\pm 2.62 \%), \mathrm{HBcAg} 18-27$ (27.75 $\pm 2.40 \%)$, and PBS (37.98 \pm $2.20 \%)(\mathrm{P}<0.01)$.

\subsection{CTP-HBcAg18-27-Tapasin Enhanced the} CD8+T Cell Response Through Regulating Phosphatidylinositol 3-kinase (PI3K)/Akt Signaling Pathway

The above results suggested that CTP-HBcAg1827-Tapasin would decrease apoptosis of $\mathrm{CD}^{+} \mathrm{T}$ cells.
Next, we investigated the activity of PI3K/Akt signaling pathway in all groups. We further analyzed the PI3K, mTOR, and Akt expression in different groups in vitro. The expression of PI3K mTOR, and Akt mRNA were detected by RT-PCR and the phosphorylation proteins were detected by western blot. The results revealed that expression of PI3K, mTOR, Akt mRNA, and PI3K PAkt and P-mTOR proteins were significantly upregulated in CTP-HBcAg18-27-Tapasin group in comparison to CTP-HBcAg18-27, HbcAg18-27-Tapasin, HbcAg18-27, and PBS groups (Figure 4).

Figure 3. The Apoptosis of CD8 ${ }^{+}$T Cells in T Cells Analyzed by Flow Cytometry
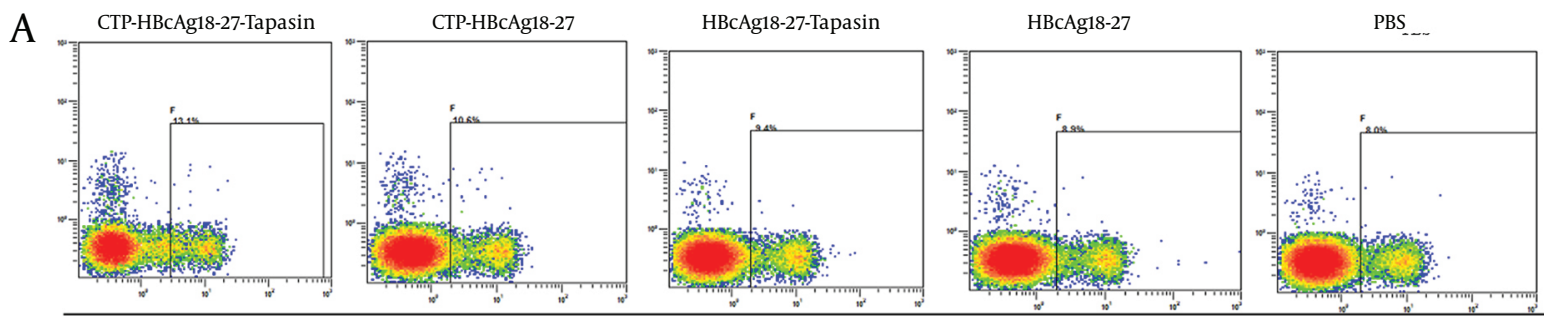

CD8 $\alpha-\mathrm{APC}$
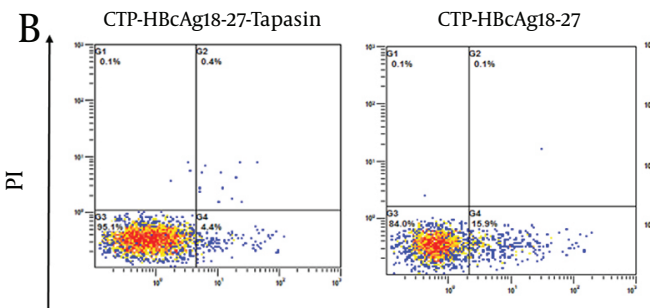

HBcAg18-27-Tapasin
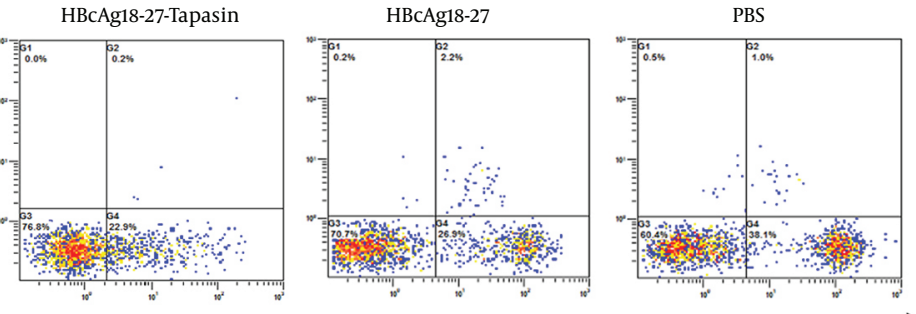

Annexin V-FITC

C

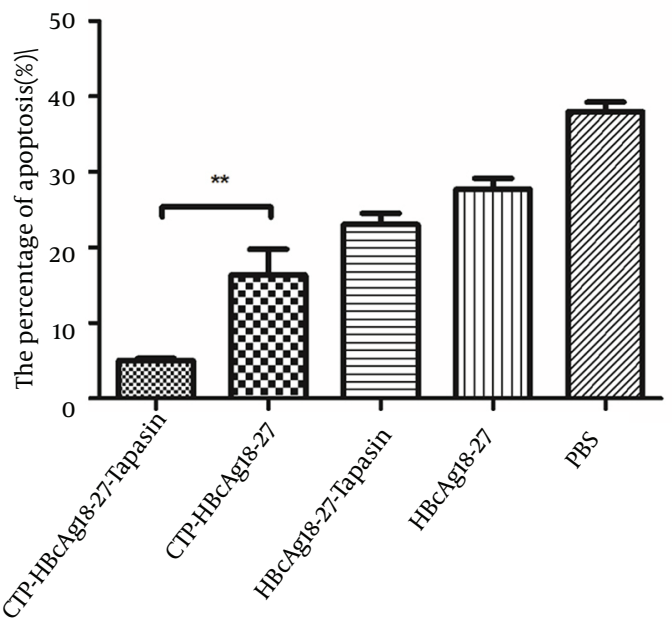

The whole cell population was stained three times with fluorescent material labeled using CD8 $\alpha$-APC antibody (A), Annexin V-FITC, and PI (B), and then counted and analyzed by flow cytometry. Significant lower percentages of apoptotic CD ${ }^{+} \mathrm{T}$ cells were observed in mice immunized with CTP-HBcAg1827-Tapasin. The data are the mean \pm SD from six mice per group $\left({ }^{* *} \mathrm{P}<0.01\right)$. 
A

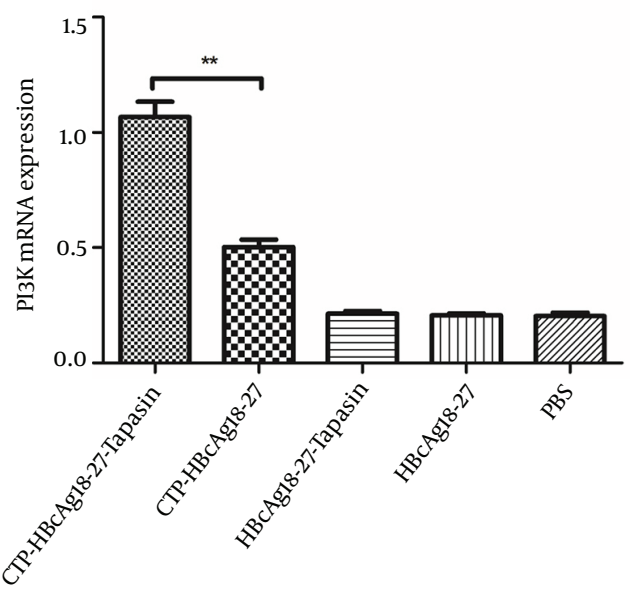

C

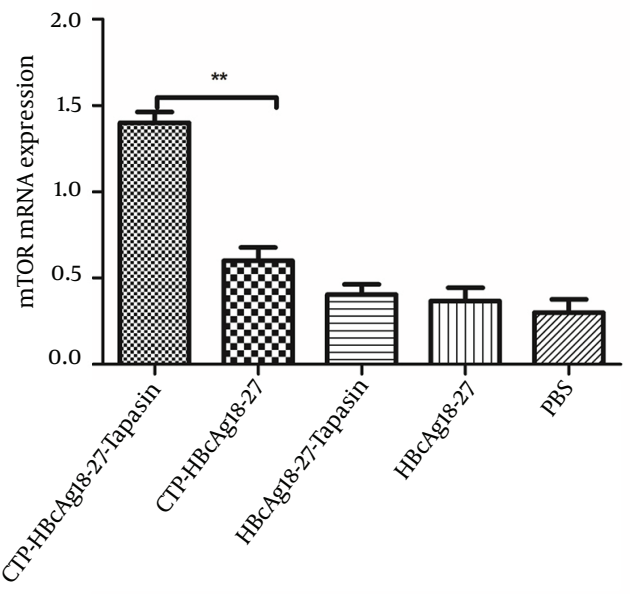

$\mathrm{E}$

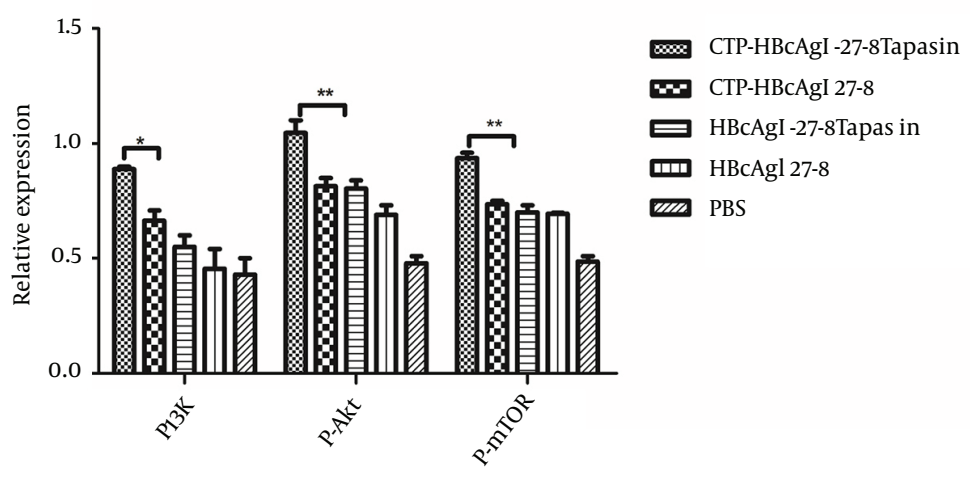

B

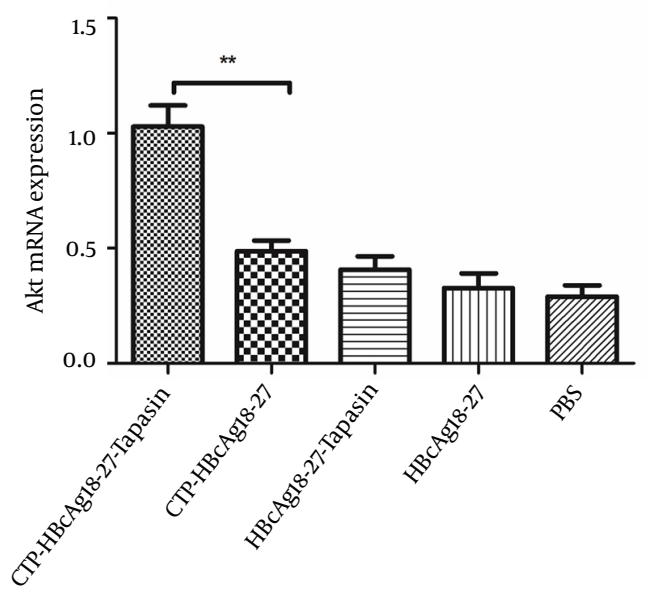

$\mathrm{D}$

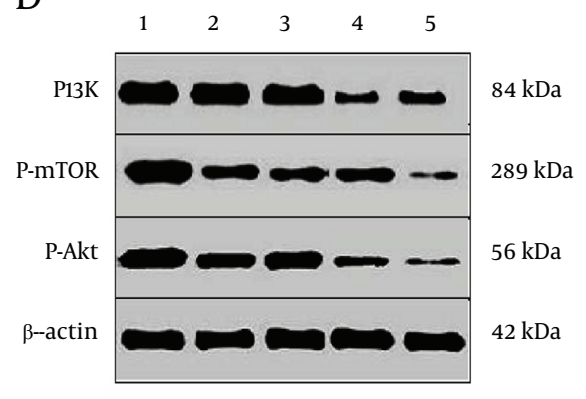

(A, B, C) The expression of PI3K, Akt, and mTOR mRNA were examined by Real-Time PCR. The above expressions were significantly upregulated in CTP-HBcAg1827-Tapasin group compared with PBS, CTP-HBcAg18-27, HBcAg18-27-Tapasin, and HBcAg18-27 groups. (D, E) Expression of PI3K, P-Akt, and P-mTOR were analyzed by Western blotting. The above proteins expressions were significantly upregulated in CTP-HBcAg18-27-Tapasin group compared with the control groups.1, CTPHBcAg18-27-Tapasin; 2, CTP-HBcAg18-27; 3, HBcAg18-27-Tapasin; 4, HBcAg18-27; 5, PBS. Data represent the mean $\pm \mathrm{SD}(\mathrm{n}=6)\left({ }^{*} \mathrm{P}<0.05,{ }^{* *} \mathrm{P}<0.01\right)$. 


\section{Discussion}

Antigen-based immune therapy (vaccine therapy) has emerged as a potential therapeutic approach for CHB patients, as it is based on the concept of viral persistence during HBV infection, it is an inadequate antiviral immune response to the viral antigens (24, 25). The HBV-specific $\mathrm{CD} 8^{+} \mathrm{T}$ cell response plays an important role in the process of HBV clearance (26). Therefore, induction of CTL responses specific to HBV represents a promising strategy to protect against HBV infection. HBV core 18-27 peptide is recognized as the most efficient agent that primes the human leukocyte antigen (HLA) class-I-restricted immune response in acutely infected patients (10). The stable assembly of the MHC class I molecules with peptides is controlled by a number of cofactors, including the peptide-loading complex. Within the peptide-loading complex, the Tapasin is a transmembrane protein that tethers empty class I molecules in the endoplasmic reticulum to the transporter associated with antigen processing, which could promote the surface expression of class I molecule and therefore improve the effectiveness of presentation of peptides to CTLs (27). In addition, it has been demonstrated that the cell-penetrating property of cytoplasmic transduction peptide (CTP) allows it to enter cells when combined with exogenous antigens and induce specific CTL responses (28-30). Thus, combining the specificity of CTL epitope (HBcAg18-27), CTP, and chaperone Tapasin may elicit robust specific HBV immune responses.

We have previously testified that the fusion protein of CTP-HBcAg18-27-Tapasin could enter cytoplasm of dendritic cells, and efficiently induce robust specific CTL response in vitro (13). In the present study, we evaluated specific CTL immune responses and the level of apoptosis of $\mathrm{CD}^{+} \mathrm{T}$ cells induced by CTP-HBcAg18-27-Tapasin fusion protein in HLA-A2 transgenic mice. At one week after the last immunization of HLA-A2 transgenic mice, the specific IFN- $\gamma^{+} \mathrm{CD} 8^{+}$T cells from CTP-HBcAg1827-Tapasin group were significantly higher than CTPHBcAg18-27, HBcAg18-27-Tapasin, HBcAg18-27, and PBS groups, which suggested that the modification of Tapasin would enhance the presentation of target antigens via intracellular delivery to $\mathrm{CD}^{+} \mathrm{T}$ cells, and induce stronger cellular immune responses. Furthermore, CTP-HBcAg18-27-Tapasin also enhanced $\mathrm{CD}^{+} \mathrm{T}$ cell activity to produce the cytokine IFN- $\gamma$, TNF- $\alpha$, and IL-2. Furthermore, the numbers of these polyfunctional triplecytokine-producing (IFN- $\gamma$, TNF- $\alpha$, and IL- 2 ) $\mathrm{CD}^{+} \mathrm{T}$ cells in CTP-HBcAg18-27-Tapasin group was higher than the control group. The inability of $\mathrm{CD}^{+} \mathrm{T}$ cell to produce three cytokines is a hallmark of functional exhaustion $(22,23)$. This result was consistent with the result of the intracellular expression of IFN- $\gamma$ in $\mathrm{CD}^{+} \mathrm{T}$ cells analyzed by flow cytometry. Taken together, these results indicated that the CTP-HBcAg18-27-Tapasin fusion protein would induce specific CTL responses.

The above results indicated that HBcAg18-27 via CTP transduction would efficiently induce $\mathrm{CD}^{+} \mathrm{T}$ cell response. However, the mechanism was not clear. During $\mathrm{CHB}$, the abundance of virus-specific $\mathrm{CD}^{+} \mathrm{T}$ cells is controlled by the balance between these cellular processes that results in a continuum of $\mathrm{T}$ cell proliferation and apoptosis (6-8). Therefore, we further observed the level of apoptosis of $\mathrm{CD}^{+} \mathrm{T}$ cells by flow cytometry. Significant lower percentages of apoptotic $\mathrm{CD}^{+} \mathrm{T}$ cells were observed in mice immunized with CTP-HBcAg18-27-Tapasin. This result indicated that CTPHBcAg18-27-Tapasin could promote $\mathrm{CD}^{+} \mathrm{T}$ cell proliferation, which was consistent with the above results. The results showed that CTP-HBcAg18-27-Tapasin would enhance the capacity of $\mathrm{CD}^{+} \mathrm{T}$ cells proliferation, cytokines release, and CTLs generation in vivo, which could efficiently activate cell-mediated immunity. Although we did not determine HBV specific CTL responses, our study showed that the enhancement of immune responses in the HLA-A2 transgenic mice induced by CTPHBcAg18-27-Tapasin had several important effects. They included significant increases of the percentages of IFN $-\gamma$ producing $\mathrm{CD}^{+} \mathrm{T}$ cells, and the numbers of these polyfunctional triple-cytokine-producing (IFN- $\gamma$, TNF- $\alpha$, and IL-2) $\mathrm{CD}^{+}{ }^{\mathrm{T}}$ cells in the spleen, the secretion of cytokine IFN- $\gamma$, IL-2, and TNF- $\alpha$; on the other hand, it significantly lowered the percentages of apoptotic $\mathrm{CD} 8^{+} \mathrm{T}$ cells. These results suggest that the acquisition of the immune responses benefits from combination of the specificity of HBcAg18-27 CTL epitope and Tapasin, and the facilitated delivery of antigens by CTP.

The phosphatidylinositol 3-kinase (PI3K)/Akt kinasesignaling axis plays an important role in a variety of cellular processes, including cytoskeletal dynamics and migration as well as survival and proliferation. For this reason, the pathway is targeted by many pathogens to reinforce or destroy focal adhesions that play an integral role in phagocytosis (31). Some studies have previously reported that PI3K is strongly activated in naive $\mathrm{T}$ cells after Ag recognition (21). During $\mathrm{CHB}$, the abundance of virus-specific $\mathrm{CD}^{+} \mathrm{T}$ cells is controlled by the balance between these cellular processes that result in a continuum of $\mathrm{T}$ cell proliferation and apoptosis (6-8). Thus, the PI3K/Akt signaling pathway might be involved in polarization toward $\mathrm{CD}^{+} \mathrm{T}$ cells. In the present study, we further analyzed the PI3K mTOR, Akt mRNA, PI3K, P-Akt, and P-mTOR proteins expression in different groups. The results revealed that expression of PI3K mTOR, Akt mRNA, and PI3K P-Akt and P-mTOR proteins were significantly upregulated in CTP-HBcAg18-27-Tapasin group compared with CTPHBcAg18-27, HBcAg18-27-Tapasin, HBcAg18-27, and PBS group. This result indicated that the CTP-HBcAg1827-Tapasin fusion protein would induce the pro-sur- 
vival activity of PI3K-Akt pathway in T cells; this was consistent with the result of the level of apoptosis of $\mathrm{CD}^{+} \mathrm{T}$ cells analyzed by flow cytometry. Therefore, the results suggested that this specific CTL activity induced by CTP-HBcAg18-27-Tapasin was related to the activity of PI3K/Akt signaling pathway in HLA-A2 transgenic mice. In conclusion, our results demonstrated that vaccination with soluble CTP-HBcAg18-27-Tapasin fusion protein would reduce apoptosis of $\mathrm{CD}^{+} \mathrm{T}$ cells, enhance the $\mathrm{CD}^{+} \mathrm{T}$ cell response, and elicit cell-mediated immunity in HLA-A2 transgenic mice, which were associated with activation of the PI3K/Akt signaling pathway.

\section{Acknowledgements}

This study was supported by grants from the National Natural Science Foundation of China (No. 31000414 and No. 81070335).

\section{Authors' Contribution}

Yong-Sheng Yu, Xiao-Hua Chen, and Yu-Yan Tang had the original idea for the study, designed the study protocol, and wrote the paper. Yi Zhang, Meng Zhuo, and Yu-Yan Tang performed the experiments. Zheng-Hao Tang and Guo-Qing Zang analyzed the data. Yong-Sheng Yu, Xiao-Hua Chen, Yu-Yan Tang, Zheng-Hao Tang, and Guo-Qing Zang wrote the final version of the paper. All authors reviewed and approved the final draft of the paper.

\section{Financial Disclosures}

All authors have no financial interests related to the material in the manuscript.

\section{Funding/Support}

This study was supported by grants from the National Natural Science Foundation of China (No. 31000414 and No. 81070335).

\section{References}

1. Ganem D, Prince AM. Hepatitis B virus infection--natural history and clinical consequences. NEngl J Med. 2004;350(11):111829.

2. Nebbia G, Peppa D, Maini MK. Hepatitis B infection: current concepts and future challenges. QJM. 2012;105(2):109-13.

3. Blackburn SD, Shin H, Haining WN, Zou T, Workman CJ, Polley A, et al. Coregulation of CD8+ T cell exhaustion by multiple inhibitory receptors during chronic viral infection. Nat Immunol. 2009;10(1):29-37.

4. Klenerman P, Hill A. T cells and viral persistence: lessons from diverse infections. Nat Immunol. 2005;6(9):873-9.

5. Ou R, Zhang M, Huang L, Moskophidis D. Control of virus-specific CD8+T-cell exhaustion and immune-mediated pathology by E3 ubiquitin ligase Cbl-b during chronic viral infection. $J$ Virol. 2008;82(7):3353-68.

6. Grayson JM, Weant AE, Holbrook BC, Hildeman D. Role of Bim in regulating CD8+ T-cell responses during chronic viral infection. J Virol. 2006;80(17):8627-38.

7. Tinoco R, Alcalde V, Yang Y, Sauer K, Zuniga EI. Cell-intrinsic transforming growth factor-beta signaling mediates virusspecific CD8+ T cell deletion and viral persistence in vivo. Immunity. 2009;31(1):145-57.

8. Wang X, Yan W, Lu Y, Chen T, Sun Y, Qin X, et al. CD4-CD8-T cells contribute to the persistence of viral hepatitis by striking a delicate balance in immune modulation. Cell Immunol. 2012;280(1):76-84.

9. Akbar SM, Chen S, Al-Mahtab M, Abe M, Hiasa Y, Onji M. Strong and multi-antigen specific immunity by hepatitis $B$ core antigen (HBcAg)-based vaccines in a murine model of chronic hepatitis $B$ : HBcAg is a candidate for a therapeutic vaccine against hepatitis B virus. Antiviral Res. 2012;96(1):59-64.

10. Chen W, Shi M, Shi F, Mao Y, Tang Z, Zhang B, et al. HBcAgpulsed dendritic cell vaccine induces Th1 polarization and production of hepatitis B virus-specific cytotoxic T lymphocytes. Hepatol Res. 2009;39(4):355-65.

11. Nanjundappa RH, Wang R, Xie Y, Umeshappa CS, Xiang J. Novel CD8+ T cell-based vaccine stimulates Gp120-specific CTL responses leading to therapeutic and long-term immunity in transgenic HLA-A2 mice. Vaccine. 2012;30(24):3519-25.

12. Purcell AW, Elliott T. Molecular machinations of the MHC-I peptide loading complex. Curr Opin Immunol. 2008;20(1):75-81.

13. Thirdborough SM, Roddick JS, Radcliffe JN, Howarth M, Stevenson FK, Elliott T. Tapasin shapes immunodominance hierarchies according to the kinetic stability of peptide-MHC class I complexes. Eur J Immunol. 2008;38(2):364-9.

14. Boulanger DS, Oliveira R, Ayers L, Prior SH, James E, Williams AP, et al. Absence of tapasin alters immunodominance against a lymphocytic choriomeningitis virus polytope. J Immunol. 2010;184(1):73-83.

15. Chen X, Lai J, Pan Q, Tang Z, Yu Y, Zang G. The delivery of HBcAg via Tat-PTD enhances specific immune response and inhibits Hepatitis B virus replication in transgenic mice. Vaccine. 2010;28(23):3913-9.

16. Chen X, Liu H, Tang Z, Yu Y, Zang G. The modification of Tapasin enhances cytotoxic T lymphocyte activity of intracellularly delivered CTL epitopes via cytoplasmic transduction peptide. Acta Biochim Biophys Sin (Shanghai). 2013;45(3):203-12.

17. Krachler AM, Woolery AR, Orth K. Manipulation of kinase signaling by bacterial pathogens. J Cell Biol. 2011;195(7):1083-92.

18. Sullivan JA, Kim EH, Plisch EH, Suresh M. FOXO3 regulates the CD8 T cell response to a chronic viral infection. J Virol. 2012;86(17):9025-34.

19. Cai N, Dai SD, Liu NN, Liu LM, Zhao N, Chen L. PI3K/AKT/mTOR signaling pathway inhibitors in proliferation of retinal pigment epithelial cells. Int J Ophthalmol. 2012;5(6):675-80.

20. Surviladze Z, Sterk RT, DeHaro SA, Ozbun MA. Cellular entry of human papillomavirus type 16 involves activation of the phosphatidylinositol 3-kinase/Akt/mTOR pathway and inhibition of autophagy. J Virol. 2013;87(5):2508-17.

21. Harriague J, Bismuth G. Imaging antigen-induced PI3K activation in T cells. Nat Immunol. 2002;3(11):1090-6.

22. Rubinstein MP, Lind NA, Purton JF, Filippou P, Best JA, McGhee PA, et al. IL-7 and IL-15 differentially regulate CD8+ T-cell subsets during contraction of the immune response. Blood. 2008;112(9):3704-12.

23. Virgin HW, Wherry EJ, Ahmed R. Redefining chronic viral infection. Cell. 2009;138(1):30-50.

24. Stoop JN, van der Molen RG, Baan CC, van der Laan LJ, Kuipers EJ, Kusters JG, et al. Regulatory T cells contribute to the impaired immune response in patients with chronic hepatitis $B$ virus infection. Hepatology. 2005;41(4):771-8.

25. Woltman AM, Op den Brouw ML, Biesta PJ, Shi CC, Janssen HL. Hepatitis B virus lacks immune activating capacity, but actively inhibits plasmacytoid dendritic cell function. PLoS One. 2011;6(1): ee15324

26. Tsai SL, Sheen IS, Chien RN, Chu CM, Huang HC, Chuang YL, et al. Activation of Th1 immunity is a common immune mechanism for the successful treatment of hepatitis B and C: tetramer assay and therapeutic implications. J Biomed Sci. 2003;10(1):120-35.

27. Chen M, Bouvier M. Analysis of interactions in a tapasin/class 
I complex provides a mechanism for peptide selection. EMBO J. 2007;26(6):1681-90.

28. Chen $\mathrm{HZ}, \mathrm{Wu} \mathrm{CP}$, Chao YC, Liu CY. Membrane penetrating peptides greatly enhance baculovirus transduction efficiency into mammalian cells. Biochem Biophys Res Commun. 2011;405(2):297-302.

29. Kim D, Jeon C, Kim JH, Kim MS, Yoon CH, Choi IS, et al. Cytoplasmic transduction peptide (CTP): new approach for the delivery of biomolecules into cytoplasm in vitro and in vivo. Exp
Cell Res. 2006;312(8):1277-88.

30. Kimura Y, Yashiroda H, Kudo T, Koitabashi S, Murata S, Kakizuka A, et al. An inhibitor of a deubiquitinating enzyme regulates ubiquitin homeostasis. Cell. 2009;137(3):549-59.

31. Fabre S, Carrette F, Chen J, Lang V, Semichon M, Denoyelle C, et al. FOXO1 regulates L-Selectin and a network of human $\mathrm{T}$ cell homing molecules downstream of phosphatidylinositol 3-kinase. J Immunol. 2008;181(5):2980-9. 